\title{
Engineering microbial consortia by division of labor
}

\author{
Garrett W. Roell', Jian Zha², Rhiannon R. Carr ${ }^{1}$, Mattheos A. Koffas², Stephen S. Fong ${ }^{3}$ and Yinjie J. Tang ${ }^{1 *}$ (D)
}

\begin{abstract}
During microbial applications, metabolic burdens can lead to a significant drop in cell performance. Novel synthetic biology tools or multi-step bioprocessing (e.g., fermentation followed by chemical conversions) are therefore needed to avoid compromised biochemical productivity from over-burdened cells. A possible solution to address metabolic burden is Division of Labor (DoL) via natural and synthetic microbial consortia. In particular, consolidated bioprocesses and metabolic cooperation for detoxification or cross feeding (e.g., vitamin C fermentation) have shown numerous successes in industrial level applications. However, distributing a metabolic pathway among proper hosts remains an engineering conundrum due to several challenges: complex subpopulation dynamics/interactions with a short time-window for stable production, suboptimal cultivation of microbial communities, proliferation of cheaters or low-producers, intermediate metabolite dilution, transport barriers between species, and breaks in metabolite channeling through biosynthesis pathways. To develop stable consortia, optimization of strain inoculations, nutritional divergence and crossing feeding, evolution of mutualistic growth, cell immobilization, and biosensors may potentially be used to control cell populations. Another opportunity is direct integration of non-bioprocesses (e.g., microbial electrosynthesis) to power cell metabolism and improve carbon efficiency. Additionally, metabolic modeling and ${ }^{13} \mathrm{C}$-metabolic flux analysis of mixed culture metabolism and cross-feeding offers a computational approach to complement experimental research for improved consortia performance.
\end{abstract}

Keywords: ${ }^{13} \mathrm{C}$-metabolic flux analysis, Cross-feeding, Metabolite channeling, Reporter protein, Subpopulations

\section{Background}

As the fields of synthetic biology have advanced, biological production has become a promising option for manufacturing various chemicals and pharmaceuticals. In principle, any compound with a defined synthesis pathway could be produced from cheap feedstock by an engineered microbial culture. Unfortunately, while recombinant microorganisms have been generated to produce some molecules, their productivity rarely reaches sufficient levels for commercialization [1]. One key barrier is metabolic burden (Fig. 1). Microbial hosts have to allocate limited resources amongst different tasks; this essential balancing act describes the key counterforce against any engineered

\footnotetext{
*Correspondence: yinjie.tang@seas.wustl.edu

${ }^{1}$ Department of Energy, Environmental and Chemical Engineering,

Washington University, Saint Louis, MO 63130, USA

Full list of author information is available at the end of the article
}

pathway [2-4]. Additionally, chemical production and intermediate accumulation will often cause cell stress due to chemical toxicity [5], and the host may consume additional ATP/NADH (e.g., use of efflux pump) [6], leading to further resource shortages [7]. The synergistic combination of metabolic burden and cell stress leads to a deep drop in microbial biosynthetic performance, termed the "metabolic cliff" [4]. When the host hovers at the edge of this cliff, even small growth/ stress perturbations can cause undesired metabolic responses and loss of production yields. To minimize such problems, Division of Labor (DoL) using microbial consortia becomes an alternative strategy [8]. Drawing inspiration from natural systems may provide one or more routes to circumvent this metabolic cliff. In higher organisms, specific metabolic tasks are partitioned off into membrane-bound organelles. Compartmentalizing different portions of a synthesis pathway can also serve to spatially separate mutually 


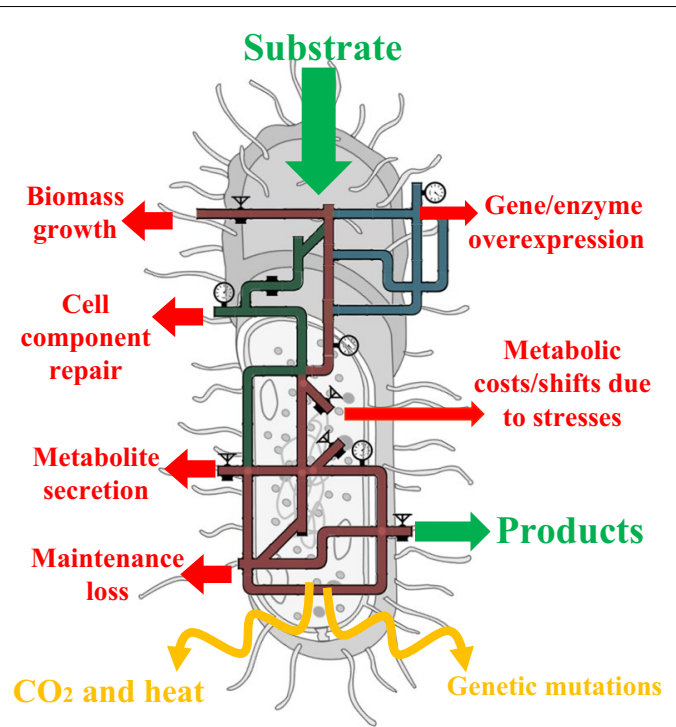

Metabolic Burdens

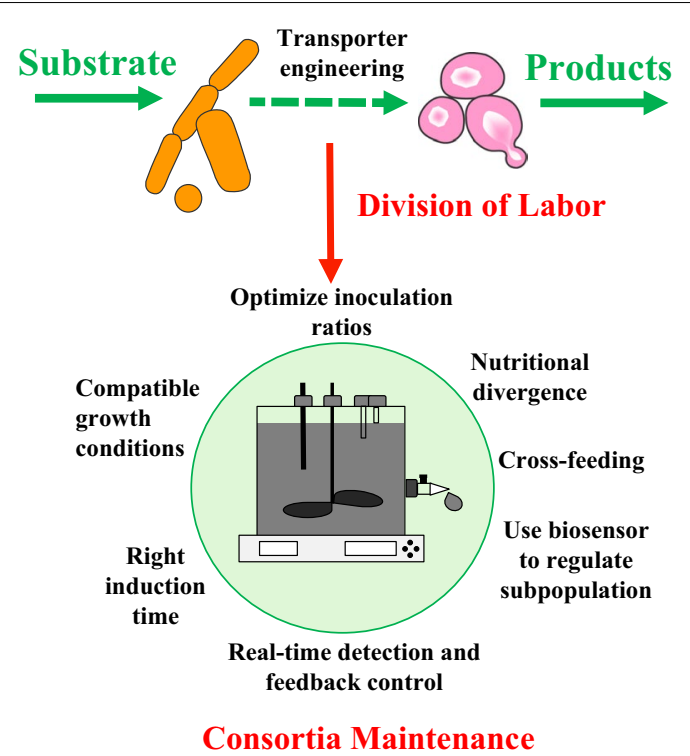

Consortia Maintenance

Fig. 1 Overview of cellular processes, metabolic burdens, and resource allocations in engineered microbial hosts (left) and the consortia maintenance (right)

incompatible steps. From an ecological perspective, different species in microbial consortia cross-feed nutrients, allowing the system to achieve diverse functions from bioremediation to biochemical production $[9,10]$. Even for genetically identical cells, multiple subpopulations can divide the biosynthesis labor or sequester toxic intermediates (as in heterocystous cyanobacteria). Extending these concepts to bioprocesses presents new opportunities for engineering pathways and controlling cell populations to maintain homeostasis (Fig. 1).

Microbial consortia can break up metabolic load among partners [11-13]. In the past, microbial consortia have been used for waste treatment, anaerobic digestion, the food/nutrient industry (e.g., dairy products, soy sauce, wines, and vitamins), as well as medical applications (e.g., gut microbiome). Recently, synthetic biology has successfully engineered co-cultures for the production of commodity chemicals (cis,cis-muconic acid) [14] as well as drugs (oxygenated taxanes) [15]. Synthetic consortia can be formed from several strains of the same species, such as engineered $E$. coli strains which together produce high-value chemicals such as flavan-3-ols [16], curcuminoids [17], and anthocyanins [18]. Current industrial applications of DoL using microbial consortia still face challenges in controlling population dynamics and optimizing productions. This review presents an overview of microbial consortia applications, limitations, and opportunities.

\section{Microbial consortia interactions, maintenance and stability}

Microbial communities are ubiquitous in natural environments and are key players in global carbon and nutrient cycles [19]. Several types of co-culture relationships are possible between two microbial species (Fig. 2) [20]. First, if two species consume different substances (i.e., nutritional divergence) and neither produces inhibitory compounds, the presence of each will not affect the other's growth; this situation is described as 'neutralism'. Second, if both species need the other to survive, which can happen when species mutually exchange required substances, or mutually remove toxins, the relationship is termed 'mutualism. As an example, a co-culture of Desulfovibrio vulgaris and Methanococcus maripaludis was developed for methane production from organic acids [19]. The paradigm for the syntrophic association is that methanogens create favorable thermodynamic conditions by scavenging hydrogen and keeping its partial pressure low, allowing the sulfate reducer to ferment carbon sources. Further, protocooperation is similar to mutualism, except that the interaction between species is beneficial to the growth rate of both populations but not required for either to live. Third, commensalism and amensalism both describe one-way interactions, where one species affects the well-being of another while remaining unaffected by its partner. In commensalism, the effect is beneficial, whereas in amensalism, growth of the affected species can be hindered due to 

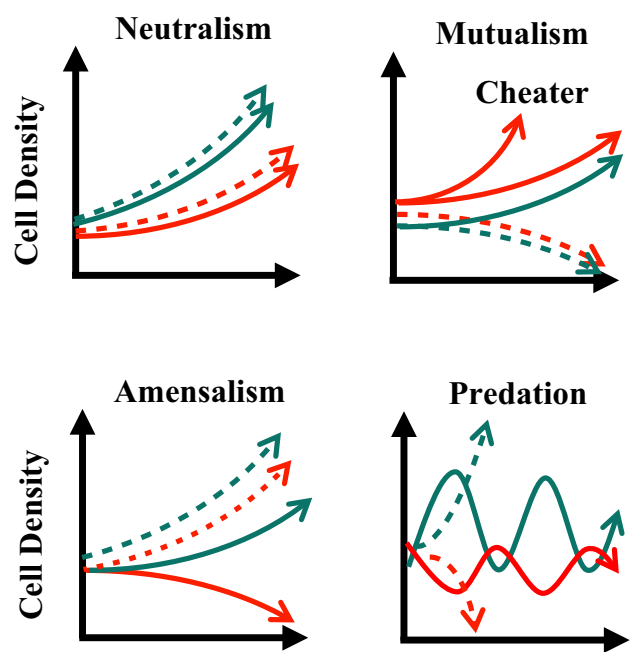
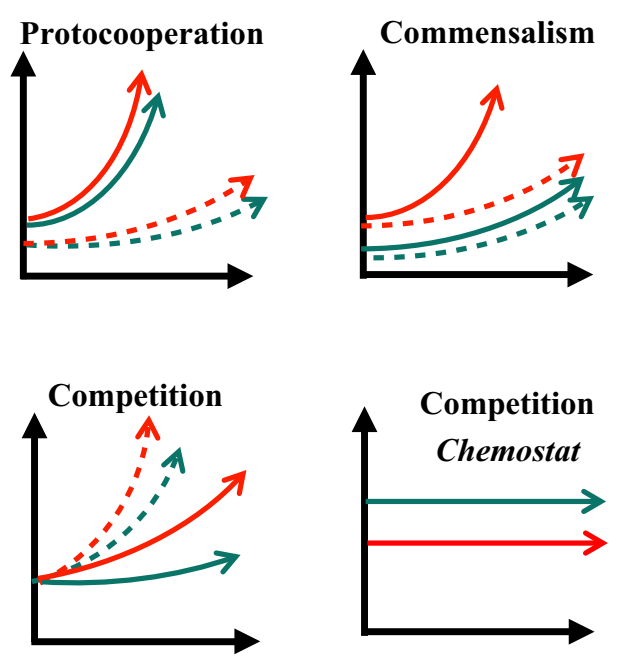

Time

Fig. 2 Interactions between two species in co-culture can have many different characters (red=species 1, green =species 2, solid=co-culture, dashed $=$ pure culture)

the production of toxic compounds from its partner. Fourth, predation (or parasitism, less observed in microbial consortia) describe scenarios where one species' growth depends on consuming the other, in which the population dynamics often show continuous oscillations. Finally, if different species compete for the same limited substrate, the faster-growing species will dominate over time. However, different species may still coexist at a stable population ratio in a chemostat culture when dilution rate and substrate concentrations are maintained at the crossover point where both species have the same growth rate [20].

Due to the complex interactions and dynamics of species within a community, consortia maintenance and stability is crucial for any successful applications (Fig. 1). Different microbes in consortia must grow in the same environment (temperature, media, $\mathrm{pH}$, and oxygen) and the growth of one species must not destroy the other members in a short period. Since the growth rates of different species or different partner strains from the same species will not be identical, one species can eventually take over the culture. To balance the subpopulations, several approaches may be employed. First, the inoculation ratios for different partners must be optimized. Second, intermittent supplementation of underdog subpopulations may elongate the period of co-cultivation. With the aid of real-time detection systems [21], optimized population composition and bioprocess parameters can be closely monitored and maintained across the fermentation by feeding the desired cultures. Third, biosensors (e.g., quorum sensing through cell-to-cell communication) may potentially be used to control cell sub-population [22]. Fourth, cell immobilization can be attempted (e.g., growing free cells of Pichia stipites and immobilized Zymomonas mobilis together for ethanol production) [23]. Fifth, coexistence partners often compete for substrates, but nutritional divergence or syntrophy (one species lives off the products of another species) can be employed to avoid substrate competition. Such concepts have been widely applied for utilization of mixed substrates or cascade biodegradation of recalcitrant feedstock. Importantly, mutualistic growth is desirable for stable consortia applications. During this cooperation, a species benefits from the waste of another, while the waste producer might also receive costly resources in return. Such mutualistic consortia can show stable performance over prolonged cultivations [19]. Moreover, in situ evolution can improve the robustness of mutualistic systems. For example, in a stable co-culture, Salmonella enterica evolved to secrete methionine, a costly amino acid, for an E. coli strain, while the E. coli evolved novel secretion of sugar to feed S. enterica [24, 25].

\section{Applications of DoL for substrate utilizations}

Microbial communities have been extensively studied for microbial ecology and waste treatment. Industrial biotechnology has applied the same principles to increase bioproduction from cheap feedstocks such as $\mathrm{CO}_{2}$ and cellulosic biomass. Synthetic consortia composed of bacteria and fungi are a particularly promising method for 
utilizing agricultural wastes to achieve waste-to-fuel/ waste-to-food processes [26].

\section{Consolidated bioprocesses}

Consolidated bioprocesses (CBP) aim to directly convert lignocellulosic biomass to biofuel. Attempts have been made to engineer a super bug that can convert cellulose to ethanol or other fuels. In one attempt, Saccharomyces cerevisiae was engineered to carry minicellulosomes to give cells the ability to simultaneously break down and ferment cellulose to ethanol. However, this engineered strain only achieved a low titer of ethanol $(\sim 1.8 \mathrm{~g} / \mathrm{L})$ [27]. In contrast, cellulolytic thermophiles, such as Clostridium thermocellum, can be a CBP platform for ethanol production, but they have low ethanol yields and resistance to genetic modifications. To overcome drawbacks associated with singleorganism CBP, synthetic co-cultures are developed, where cellulose is fed into the system and the product of interest is synthesized using two different organisms: a cellulose degradation module that secretes cellulases and a product synthesis module that consumes the resulting glucose to make the product (Fig. 3). For example, co-cultures of Clostridium thermocellum with bio-producing bacteria could be a promising approach.
Specifically, the fermentation of cellulose and cellobiose by Clostridium thermocellum and Methanobacterium thermoautotrophicum co-culture was built to produce hydrogen gas, methane, acetic acid, and ethanol [28]. More recently, co-cultures of Clostridium thermocellum and non-cellulolytic Thermoanaerobacter strains significantly improved ethanol production by 4.4 -fold compared to the monoculture [29]. CBP for butanol production from cellulosic biomass have been developed by taking advantage of the specific metabolic capacities of cellulosic Clostridium celevecrescens and butanol producer Clostridium acetobutylicum. They have achieved butanol concentration of $3.73 \mathrm{~g} / \mathrm{L}$ [30]. Another avenue is to employ fungal strains paired with genetically-engineered E. coli for isobutanol production [31]. The fungus Trichoderma reesei secretes cellulases to hydrolyze lignocellulosic biomass and $E$. coli metabolizes soluble saccharides into isobutanol (achieving titers up to $1.9 \mathrm{~g} / \mathrm{L}$ and yields up to $62 \%$ of theoretical maximum). Trichoderma reesei and Rhizopus delemar can also be coupled to convert cellulose to fumaric acid $(6.87 \mathrm{~g} / \mathrm{L}$ and $0.17 \mathrm{w} / \mathrm{w}$ yield) without supplemental cellulase enzymes [32]. In another example, a strategy was developed by constructing a cell-surface displayed consortium using two engineered yeast that heterologously

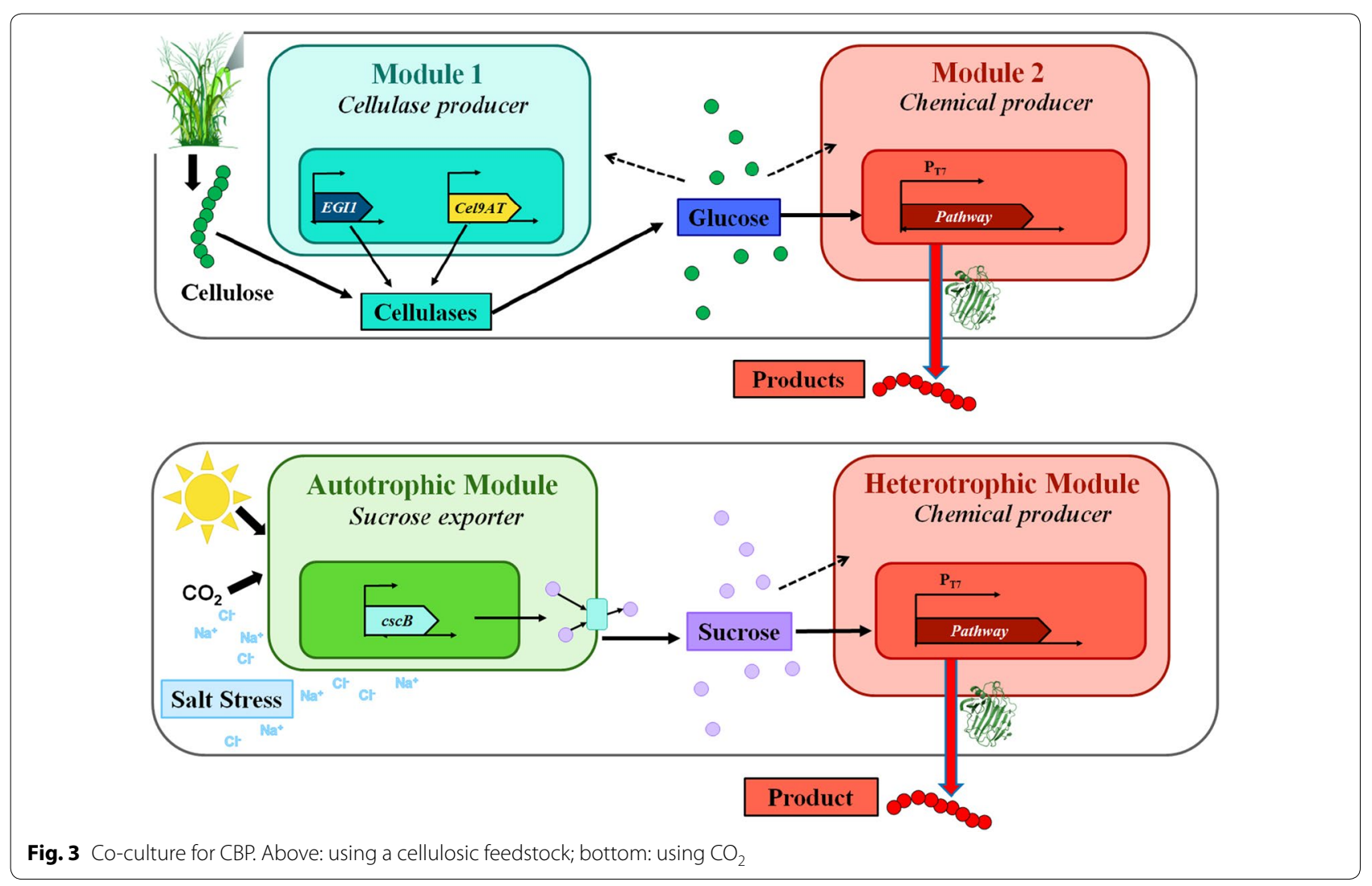


expressed functional lignocellulolytic enzymes to convert pretreated corn stover to ethanol [33].

\section{Mixed sugar fermentations}

For cellulosic feedstock, xylose derived from the hemicellulose component cannot be used by model yeast platforms. To address this, a xylose-fed co-culture of $E$. coli and Saccharomyces cerevisiae has been developed, wherein $E$. coli (which can naturally use xylose) generate acetate as waste product; $S$. cerevisiae consumes the acetate and reduces its inhibition of $E$. coli growth [15]. Similarly, consortia with different engineered S. cerevisiae strains can ferment glucose-xylose-arabinose mixtures [34].

\section{C1 carbon utilizations}

Consortia can serve as platforms for utilization of $\mathrm{C} 1$ substrates (such as methane, $\mathrm{CO}_{2}$, and $\mathrm{CO}$ ). For example, Citrobacter amalonaticus can assist Sporomusa ovata to produce acetic acid from carbon monoxide [35]. Moreover, methane is an abundant feedstock from anaerobic digestion or oil reservoirs. Archaeal-bacterial symbiosis could couple methane oxidation with sulphate reduction in gas-hydrate-rich sediments [36], which makes the anaerobic conversion of methane into bio-products possible. Among $\mathrm{C} 1$ substrates, biosequestration of $\mathrm{CO}_{2}$ has great potential. Algae, including both prokaryotic cyanobacteria and eukaryotic microphytes, can fix inorganic carbon for bio-production. However, algal growth and genetic tool development have lagged far behind that of classic heterotrophic hosts like E. coli and S. cerevisiae [37]. It is possible, however, for the autotrophy of microalgae to be exploited more indirectly, in a modular system that supplies organic carbon for a chemicalproducing heterotrophic host (Fig. 3). Such modularity (algae-bacteria system) has been laid in the development of a sucrose-exporting strain of the cyanobacteria Synechococcus elongatus PCC 7942, which heterologously expresses the proton-sucrose symporter $\csc B$. Under conditions of osmotic stress, this $\csc B^{+}$strain irreversibly secretes up to $85 \%$ of fixed carbon as sucrose [38]. This cyanobacterial host has been utilized in synthetic microbial consortia with a variety of microbes, including model strains (E. coli, Bacillus subtilis, and S. cerevisiae), soil bacteria (Pseudomonas putida, Halomonas boliviensis), and diazotrophs (Azotobacter vinelandii) to produce a variety of products without additional sugar input [3942]. Autotroph-heterotroph coupling is not limited to cyanobacteria-binary culturing of the eukaryotic algae Haematococcus pluvialis with $B$. subtilis demonstrated a coupling of oxygenic photosynthesis with respiration, such that neither external $\mathrm{CO}_{2}$ nor $\mathrm{O}_{2}$ supplies were necessary, suggesting that some of the overhead costs associated with autotrophs could be reduced in a properly-configured production system [43]. Similarly, cocultures of eukaryotic algae Chlorella minutissima with E. coli can increase neutral lipid production and improve biodiesel quality [44]. These studies facilitate the application of $\mathrm{CO}_{2}$-to-fuel technologies.

\section{Nutrient crossing feeding using companion strains}

Interacting partners can be designed to maintain stable interactions through detoxifying inhibitory substances. For instance, Dehalococcoides mccartyi is an important bacterium involved in the bioremediation of chlorinated solvents, but its incomplete Wood-Ljungdahl pathway generates toxic $\mathrm{CO}$ [45]. Co-cultures with CO-consuming bacterium Desulfovibrio vulgaris Hildenborough as the companion can significantly support $D$. mccartyi growth and TCE degradation ability. In another example, L-ascorbic acid (vitamin C) is produced via a twostep fermentation process to reduce costs and increase product quality. In the second step, a mixed fermentation consisting of a production strain (Ketogulonicigenium vulgare) and a companion strain (e.g. Bacillus spp.) is used to convert L-sorbose to 2-keto-L-gulonic acid and ascorbic acid [46]. The interaction mechanisms between $K$. vulgare and the companion strain have been studied, and it was found that the companion strain secreted metabolites to support $K$. vulgare growth (including proteins, some amino acids, and other unknown substances) while $K$. vulgare can also inhibit growth of the companion strain by lysis of companion cells (thus both mutualism and amensalism exist in this artificial ecosystem).

\section{Division of long-step biosynthesis pathways among different species}

The introduction of long-step biosynthetic pathways in a single bacterial strain can cause severe metabolic burden due to the overwhelming consumption of cellular build blocks and ATP for enzyme synthesis. In contrast, dividing the pathway into multiple strains in a synthetic consortium can split the overall metabolic burden among the constituent strains, with each strain bearing significantly lower stresses [21] (Fig. 4). Such a strategy has been adopted for the expression of an entire anthocyanin pathway from tyrosine in a four-strain consortium, employing an efficient tyrosine-producing strain as the first node strain [18]. This artificial microbial community gave rise to the direct production of $\sim 10 \mathrm{mg} / \mathrm{L}$ pelargonidin 3-O-glucoside from glucose, which is functionally impossible via monoculture. Another advantage of microbial consortia is that the use of multiple species in one pathway can ensure that all genes are properly expressed [47]. In the microbial production of natural products, some genes with plant or other eukaryotic origins may not be 
Monoculture with the long-step biosynthesis pathway

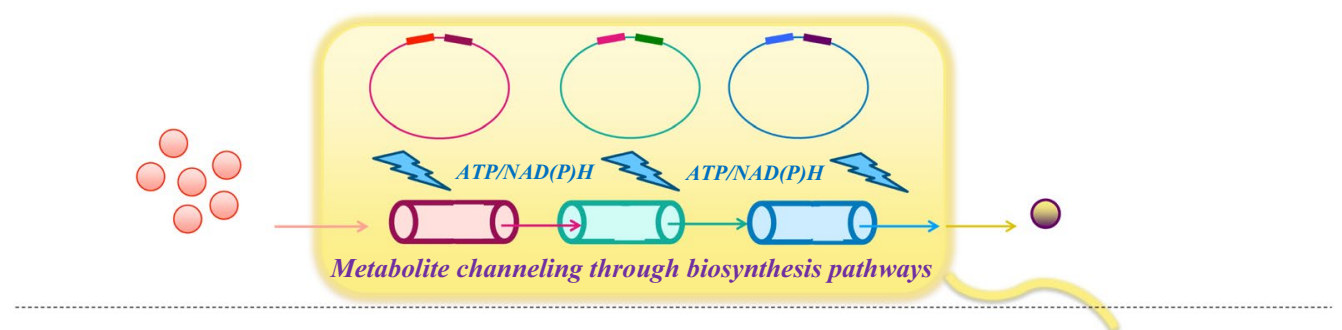

DoL: Mixed culture for cascade conversions

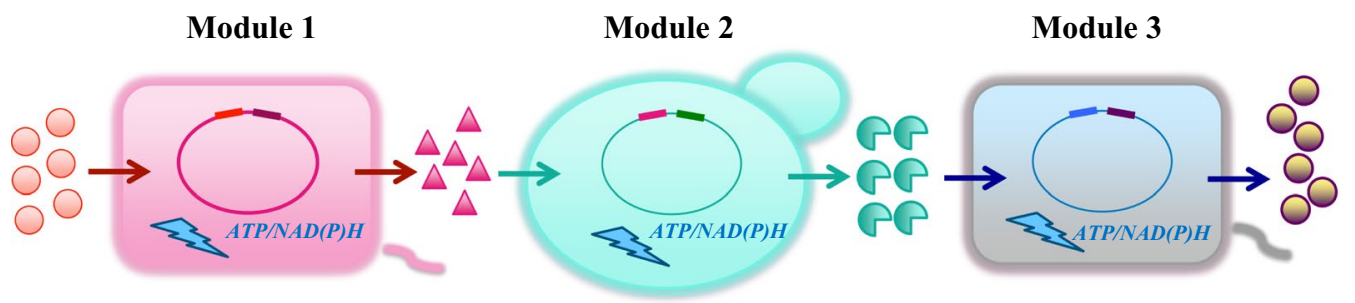

Fig. 4 Schematic comparison of a microbial consortium and a monoculture in the expression of a challenging metabolic pathway. Microbial consortia-based division of metabolic pathways into multiple strains can reduce metabolic burden imposed on each individual constituent strain. Also, specific modules can be assigned to suitable strains for optimal enzyme expression (different colors of cells represent different host strains). However, DoL may break innate metabolite channeling and dilute intermediate metabolites in the biosynthesis pathways

functionally expressed due to codon bias, improper protein folding, or the lack of post-translational modifications [48]. Additionally, the effective operation of many modules relies on a sufficient supply of cofactors (such as $\mathrm{NAD}^{+} / \mathrm{NADH}$ and $\mathrm{NADP}^{+} / \mathrm{NADPH}$ ) or active building blocks (such as acetyl-CoA and malonyl-CoA). While it is difficult to maximize every module simultaneously in a single strain, DoL can facilitate the functional expression of the complete pathway. With all the modules fully potentiated and the inter-module interference minimized by spatial segregation, DoL greatly improves pathway efficiency. A successful demonstration of such a method is the high-titer production of oxygenated taxanes in a S. cerevisiae-E. coli consortium. S. cerevisiae was used to express a P450 taxadiene hydroxylase (which could not be expressed functionally in E. coli), and E. coli was engineered for efficient taxadiene production. This coculture generated $33 \mathrm{mg} / \mathrm{L}$ oxygenated taxanes, which were undetectable in either $E$. coli or yeast monoculture harboring the entire pathway [15]. Division of challenging pathways in optimal constituent strains in a microbial consortium is feasible for the bioproduction of various complex natural compounds such as flavonoids, isoprenoids and alkaloids. Moreover, DoL potentially simplifies optimization of these metabolic pathways. For a monoculture, construction of an efficient heterologous pathway usually involves adjustment of pathway components, such as promoters, ribosome binding sites, terminators, and vectors, followed by the Design-Build-Test cycle [49], which is labor-intensive and cost-ineffective. In comparison, the optimization of microbial consortia is somewhat easier, and is achieved simply by changing the ratio of the constituent strains [16]. Theoretically, the alteration of the culture component can be performed at any stage of the fermentation process by supplementing the desired subcultures.

\section{Limitations of DoL and future directions}

Culturing microbial workhorses as a community faces several key limitations. First, many microbial community systems are dynamic and cannot achieve long-term production stability. Applications of consortia systems require identifying both an optimal inoculation ratio and an ideal product induction time. Nevertheless, the stability of the co-culture is expected to decrease in large volume and long-term fermentation processes due to spatiotemporal dynamics inside of reactors [24], incompatible growth requirements (e.g., the cross-feeding is insufficient to sustain net growth of both partners), or metabolite dilutions [50]. Second, two different species in co-cultures have to grow under suboptimal or compromised conditions. For example, to stabilize a co-culture of E. coli and S. cerevisiae, S. cerevisiae must be grown with acetate to avoid producing toxic ethanol [15]. Since acetate is not a favorable carbon source for yeast, the final bio-manufacturing capability is impaired. 
Third, metabolite transport can be an issue if a pathway is divided, such that the first product must be transported into the second host for further conversion. If the first product (e.g., a phosphorylated molecule) is unstable or unable to cross the cell membrane, or the second host lacks a transporter to uptake the precursor from the first host, the DoL will fail. Engineering effective transporters and efflux systems is not easy and may also add metabolic burdens [51]. Furthermore, long-step biosynthesis pathways often require proximity channeling to overcome in vivo diffusion limitation, metabolite loss, and thermodynamic barriers. DoL of a biosynthesis pathway between two different species may break innate channels and dilute intermediate concentrations, which unavoidably hinders biosynthesis efficiency [52] (Fig. 4). Fourth, microbial species may naturally evolve into distinct phenotypic subpopulations (e.g., E. coli may form two distinct cell populations with acetate cross-feeding) [53]. In mutualistic consortia, cheaters (individual cells that do not help the other species) can be observed-cheaters face less metabolic burden and can achieve a faster growth rate, but when they become dominant, the coculture will be unable to survive [54]. For example, in a CBP-based isobutanol fermentation, fungus T. reesei and engineered $E$. coli consortia form stable cooperatorcheater dynamics [31]. Interestingly, even in a pure culture of synthetic hosts, nongenetic cell-to-cell variations can be high, allowing low-production cheaters to eventually become dominant [55].

Current community-based biomanufacturing mainly leverages natural systems and focuses on utilization of recalcitrant or cheap feedstock. In these desired platforms, metabolic interactions (e.g., cross feeding) can be robustly evolved. Still, there are few industrial applications based on the division of biosynthesis pathways among different species. For future DoL developments, a few approaches are promising. First, biosensors can be used to monitor and control cell sub-populations. In addition to quorum sensing for population monitoring, an RNA riboswitch-based biosensor module has been developed to provide real-time screening for overproducing cells in co-cultures [56]. Biosensor-regulator systems may be used for in vivo population quality control to continuously select for high-performing variants by suppressing the growth of cheaters [55]. Second, state-of-the-art bioreactor operations with the aid of readily-available high-throughput tools and real-time detection systems allow for feedback control/optimization of population composition (supplements of desired culture) and bioprocess parameters (e.g., substrate feeding) to maintain co-culture fermentation for stable and efficient biomanufacturing [21]. Third, the integration of non-bioprocess methods (Chem + electrobio) is another future direction [57]. Microbial electrosynthesis is a novel technology which combines metabolic engineering and electrochemistry within a biological reactor and has the ability to achieve $100 \%$ carbon efficiency by generating reducing equivalents (in the form of $\mathrm{NADH}$ ) from electrons [58]. This type of electrochemical bioreactor may also capture and reduce $\mathrm{CO}_{2}$, thus moving carbon into the desired metabolic pathway. A similar concept has been used to combine hybrid inorganic and biological organisms for solar-to-chemical production $[59,60]$.

\section{Analysis and modeling of consortia and metabolic interactions between species}

There are many tools being developed to study consortia and DoL applications. Kinetic modeling is the most common method to analyze nutrient cross-feeding and microbial interactions [20]. For example, E. coli and photoheterotrophic Rhodopseudomonas palustris crossfeed carbon (organic acids) and nitrogen (ammonium) to form a stable co-culture. Monod-based differential equations have been developed to reveal fermentation conditions for stabilizing mutualism [61, 62]. The model results indicate that organic acids from $E$. coli can be inhibitory and thus decrease carbon utilization efficiency and population equilibrium. For molecular-level understanding, functional genomics and high-quality metabolic modeling are needed to decipher cellular regulatory networks and integrated functions. For consortia, functional analysis can rely on typical microbiome tools, including targeted cell population analysis (such as $16 \mathrm{~S}$ rRNA), metagenomic sequencing (DNA is recovered in an untargeted manner), metatranscriptomics (study the gene expression of microbial communities), and metaproteomics (study proteins expressed by microbiota to gain insight into functional potential). Microfluidic cell sorting can assist in the separation of individual species to study their DNA-RNA-Protein profiles under a community system. Moreover, measurement of metabolites within community systems can monitor the global community outcome and provide a snapshot of the general physiological state of microbes $[46,63]$.

To date, methods applicable for analyzing metabolic fluxes between two species (especially for two engineered strains derived from the same parent strain) are still rare. Currently, genome-scale models (GSMs) predict the feasible fluxes based on stoichiometry of the metabolic reactions, cellular objective functions, and constraints [64-68]. Co-culture/tri-culture stoichiometric models have been developed to analyze compositions of microbial communities for the biogas process [69]. Such models can potentially combine gene expression data or translational profiles as reaction constraints to capture information passage from DNA $\rightarrow$ RNA $\rightarrow$ proteins 
[70]. For example, GSM and metatranscriptomic data are used to study the uncultured bacterial symbiont "Candidatus Endobugula sertula" and identify their metabolic deficiencies [71]. Flux balance analysis (FBA) using reconstructed metabolic networks was attempted for the syntrophic Desulfovibrio vulgaris/Methanococcus maripaludis chemostat system [19]. The modeling results were compared to their continuous bioreactor data on lactate cultures in the absence of sulfate, and were found to accurately predict ecologically-relevant characteristics and growth parameters of bacterial communities. FBA, however, requires the assumption of a pseudo steadystate system. To capture complex dynamics in time and space of microbial communities, dynamic flux balance analysis (dFBA) is employed. In a co-culture model for cellulolytic Clostridium cellulolyticum and the solventogenic Clostridium acetobutylicum, an adapted dFBA was used where the key parameters required are $\mathrm{k}_{\mathrm{m}}$ and $\mathrm{V}_{\max }$ for cellulose solubilization and cell metabolism, as well as starting biomass concentrations for the two co-culture strains to set the input conditions for the growth dynamics [72]. dFBA can simulate the time-step interaction of the individual steady state models, and measurements of key central metabolites can be used to test the accuracy of the co-culture simulation. In another example, dFBA for multiple organisms was developed to address whether ecosystem-level behavior of structured communities can be predicted. The modeling framework with experimentally-confirmed species ratio data simulated the community behavior and metabolic interplay among two or three species inside of a colony [24]. This result can be integrated with economic analysis for a priori estimation of synthetic co-culture performance using cellulosic sugars and flue gas [73]. In parallel, new algorithms are proposed for designing synthetic microbial communities with desired features [74, 75]. Recently, the constraintbased modeling approach was applied to the human gut microbiome, resulting in a collection of 773 GSMs for gut-associated microorganisms [76]. In addition to the computational advances for modeling multi-species systems, other research has sought to expand the depth of modeling to include additional molecular details. An example of these computational advances is a modularized approach using multiple mathematical approaches to model the whole cell function of Mycoplasma genitalium [77]. Another significant study was the implementation of transcriptional and translational machinery into constraint-based models to predict gene expression levels [78]. Together, advances in single organism models and approaches for modeling microbial consortia will enable improved understanding of consortia dynamics and the ability to prospectively design and control consortia function.
${ }^{13} \mathrm{C}$-metabolic flux analysis (MFA) can measure intracellular carbon and energy fluxes. As a simple approach, MFA can treat co-culture as a single system and determine the bulk fluxomes of this system using traditional ${ }^{13} \mathrm{C}$-MFA approaches via labeling in protein-derived amino acids. This bulk MFA only describes the system as a whole, without metabolic exchanges. The full separation of metabolites from individual species is required to reveal subpopulation fluxes and interactions between two species. Separation of subpopulation is technologically difficult, even with fast cell-sorting. However, a "reporter protein" synthesized by a specific cell type can store ${ }^{13} \mathrm{C}$-fingerprints to investigate the microbial community. For mixed culture MFA, instead of detecting the ${ }^{13} \mathrm{C}$ labeling patterns in amino acids from the total cellular protein, ${ }^{13} \mathrm{C}$ patterns in amino acids from the reporter proteins can provide labeling information for each individual species. For example, photosystem I is a reliable reporter protein to probe symbiotic interactions in algae-heterotrophic bacterial communities [79]. Specifically, photosystem I proteins are abundant in algae and these proteins form large complexes that can be separated from the culture via ultracentrifuge and used as the reporter to study algae-bacteria cultures. For engineered species, the reporter protein can be overexpressed with a His-tag, allowing affinity purification from bulk culture $[79,80]$. For example, the recombinant fusion glutathione S-transferase and green fluorescent protein can be used as the reporters to study mixed-culture of $E$. coli mutants. The reporter method quantitatively resolved the expected mutant-specific phenotypes down to subpopulation fractions of $\sim 1 \%$ [81]. Similarly, peptide-based ${ }^{13} \mathrm{C}$-MFA has been developed to measure intracellular metabolic fluxes and inter-species metabolite exchange for complex microbial communities. Peptide identity and labeling patterns can be obtained in a high-throughput manner from modern proteomics techniques, which can recover metabolic fluxes in the same way as through the standard amino acid-based ${ }^{13} \mathrm{C}$-MFA [82]. Another approach for co-culture ${ }^{13} \mathrm{C}$-MFA is proposed by the Antoniewicz group, which determines metabolic flux distributions without the need for physical separation of cells or species-specific products via measurement of isotopic labeling of total biomass and elegant mathematical calculations. This approach can simulate both fluxes and the relative population size of each species in a mixed culture as well as inter-species metabolite exchange [83].

\section{Conclusion}

Division of labor via mixed cultures can theoretically reduce metabolic burden to enable a system to utilize recalcitrant feedstock or generate products which require long-step heterologous pathways. However, microbial 
consortia represent complex and dynamic systems that are difficult to operate. Community based strain engineering, metabolic modeling, and flux analysis tools are rapidly developed recently, which may facilitate the wide application of DoL concept for biomanufacturing.

\section{Authors' contributions}

GWR, RRC and YJT wrote introduction, consortia interactions, and DoL applications for feedstock utilizations. JZ and MAK wrote DoL for developing synthetic pathways. SSF and YJT wrote modeling section. All authors read and approved the final manuscript.

\section{Author details}

${ }^{1}$ Department of Energy, Environmental and Chemical Engineering, Washington University, Saint Louis, MO 63130, USA. ${ }^{2}$ Department of Chemical and Biological Engineering, Rensselaer Polytechnic Institute, 110 Eighth Street, Troy, NY 12180, USA. ${ }^{3}$ Department of Chemical and Life Science Engineering, Virginia Commonwealth University, Richmond, VA 23284, USA.

\section{Competing interests}

The authors declare that they have no competing interests.

\section{Availability of data and materials}

Not applicable.

\section{Consent for publication}

Not applicable.

\section{Funding}

This work is supported by NSF (MCB 1616619) and DOE (DESC0018324).

\section{Publisher's Note}

Springer Nature remains neutral with regard to jurisdictional claims in published maps and institutional affiliations.

Received: 31 May 2018 Accepted: 31 January 2019

Published online: 08 February 2019

\section{References}

1. Czajka J, Wang Q, Wang Y, Tang YJ. Synthetic biology for manufacturing chemicals: constraints drive the use of non-conventional microbial platforms. Appl Microbiol Biotechnol. 2017;101:7427-34.

2. Glick BR. Metabolic load and heterologous gene expression. Biotechnol Adv. 1995;13:247-61.

3. Poust S, Hagen A, Katz L, Keasling JD. Narrowing the gap between the promise and reality of polyketide synthases as a synthetic biology platform. Curr Opin Biotechnol. 2014;30:32-9.

4. Wu SG, He L, Wang Q, Tang YJ. An ancient Chinese wisdom for metabolic engineering: Yin-Yang. Microb Cell Fact. 2015;14:39.

5. Bhan N, Xu P, Koffas MAG. Pathway and protein engineering approaches to produce novel and commodity small molecules. Curr Opin Biotechnol. 2013;24:1137-43.

6. Hoehler TM, Jorgensen BB. Microbial life under extreme energy limitation. Nat Rev Microbiol. 2013;11:83-94.

7. He L, Xiu Y, Jones JA, Baidoo EEK, Keasling JD, Tang YJ, Koffas MAG. Deciphering flux adjustments of engineered $E$. coli cells during fermentation with changing growth conditions. Metabo Eng. 2017;39:247-56.

8. Dong P, Maddali MV, Srimani JK, Thelot F, Nevins JR, Mathey-Prevot B, You L. Division of labour between Myc and G1 cyclins in cell cycle commitment and pace control. Nat Commun. 2014;5:4750.

9. Saleski T, Tan JY, Lin XN. Dissecting the ecology of microbes using a systems toolbox. Cell Syst. 2017;5:442-4.

10. Jones JA, Toparlak ÖD, Koffas MAG. Metabolic pathway balancing and its role in the production of biofuels and chemicals. Curr Opin Biotechnol. 2015;33:52-9.
11. de Lima Brossi MJ, Jiménez DJ, Cortes-Tolalpa L, van Elsas JD. Soil-derived microbial consortia enriched with different plant biomass reveal distinct players acting in lignocellulose degradation. Microb Ecol. 2016;71:616-27.

12. Hanly TJ, Henson MA. Dynamic flux balance modeling of microbial cocultures for efficient batch fermentation of glucose and xylose mixtures. Biotechnol Bioeng. 2011;108:376-85.

13. Biliouris K, Babson D, Schmidt-Dannert C, Kaznessis YN. Stochastic simulations of a synthetic bacteria-yeast ecosystem. BMC Syst Biol. 2012;6:58.

14. Zhang H, Pereira B, Li Z, Stephanopoulos G. Engineering Escherichia coli coculture systems for the production of biochemical products. Proc Natl Acad Sci USA. 2015;112:8266-71.

15. Zhou K, Qiao K, Edgar S, Stephanopoulos G. Distributing a metabolic pathway among a microbial consortium enhances production of natural products. Nat Biotechnol. 2015;33:377-83.

16. Jones JA, Vernacchio VR, Sinkoe AL, Collins SM, Ibrahim MHA, Lachance DM, Hahn J, Koffas MAG. Experimental and computational optimization of an Escherichia coli co-culture for the efficient production of flavonoids. Metab Eng. 2016:35:55-63.

17. Fang Z, Jones JA, Zhou J, Koffas MAG. Engineering Escherichia coli co-cultures for production of curcuminoids from glucose. Biotechnol J. 2017; 13:e1700576.

18. Jones JA, Vernacchio VR, Collins SM, Shirke AN, Xiu Y, Englaender JA, Cress BF, McCutcheon CC, Linhardt RJ, Gross RA, Koffas MAG. Complete biosynthesis of anthocyanins using E. coli polycultures. MBio. 2017;8:e0621-17.

19. Stolyar S, Van Dien S, Hillesland KL, Pinel N, Lie TJ, Leigh JA, Stahl DA Metabolic modeling of a mutualistic microbial community. Mol Syst Biol. 2007;3:92.

20. Shuler ML, Kargi F, DeLisa M. Bioprocess engineering: basic concepts. 3rd ed. NJ: Prentice Hall Englewood Cliffs; 2017.

21. Zhang H, Wang X. Modular co-culture engineering, a new approach for metabolic engineering. Metab Eng. 2016:37:114-21.

22. Carbonell X, Corchero JL, Cubarsí R, Vila P, Villaverde A. Control of Escherichia coli growth rate through cell density. Microbiol Res. 2002;157:257-65.

23. Fu N, Peiris P, Markham J, Bavor J. A novel co-culture process with Zymomonas mobilis and Pichia stipitis for efficient ethanol production on glucose/xylose mixtures. Enzyme Microb Technol. 2009;45:210-7.

24. Harcombe WR, RiehI WJ, Dukovski I, Granger BR, Betts A, Lang AH, Bonilla G, Kar A, Leiby N, Mehta P, Marx CJ, Segrè D. Metabolic resource allocation in individual microbes determines ecosystem interactions and spatial dynamics. Cell Rep. 2014;7:1104-15.

25. Harcombe WR, Chacón JM, Adamowicz EM, Chubiz LM, Marx CJ. Evolution of bidirectional costly mutualism from byproduct consumption. Proc Natl Acad Sci. 2018;115:12000

26. Cortes-Tolalpa L, Salles JF, van Elsas JD. Bacterial synergism in lignocellulose biomass degradation-complementary roles of degraders as influenced by complexity of the carbon source. Front Microbiol. 2017;8:1628.

27. Wen F, Sun J, Zhao H. Yeast surface display of trifunctional minicellulosomes for simultaneous saccharification and fermentation of cellulose to ethanol. Appl Environ Microbiol. 2010;76:1251-60.

28. Weimer PJ, Zeikus JG. Fermentation of cellulose and cellobiose by Clostridium thermocellum in the absence of Methanobacterium thermoautotrophicum. Appl Environ Microbiol. 1977;33:289-97.

29. He Q, Hemme CL, Jiang H, He Z, Zhou J. Mechanisms of enhanced cellulosic bioethanol fermentation by co-cultivation of Clostridium and Thermoanaerobacter spp. Bioresour Technol. 2011;102:9586-92.

30. Wang Z, Cao G, Zheng J, Fu D, Song J, Zhang J, Zhao L, Yang Q. Developing a mesophilic co-culture for direct conversion of cellulose to butanol in consolidated bioprocess. Biotechnol Biofuels. 2015;8:84.

31. Minty JJ, Singer ME, Scholz SA, Bae CH, Ahn JH, Foster CE, Liao JC, Lin XN. Design and characterization of synthetic fungal-bacterial consortia for direct production of isobutanol from cellulosic biomass. Proc Natl Acad Sci USA. 2013;110:14592-7.

32. Scholz SA, Graves I, Minty JJ, Lin XN. Production of cellulosic organic acids via synthetic fungal consortia. Biotechnol Bioeng. 2018;115:1096-100.

33. Chen L, Du JL, Zhan YJ, Li JA, Zuo RR, Tian S. Consolidated bioprocessing for cellulosic ethanol conversion by cellulase-xylanase cell-surfaced yeast consortium. Prep Biochem Biotechnol. 2018;48:653-61.

34. Verhoeven MD, de Valk SC, Daran J-MG, van Maris AJA, Pronk JT. Fermentation of glucose-xylose-arabinose mixtures by a synthetic consortium of single-sugar-fermenting Saccharomyces cerevisiae strains. FEMS Yeast Res. 2018;18:foy075. 
35. Lee CR, Kim C, Song YE, Im H, Oh YK, Park S, Kim JR. Co-culture-based biological carbon monoxide conversion by Citrobacter amalonaticus Y19 and Sporomusa ovata via a reducing-equivalent transfer mediator. Bioresour Technol. 2018;259:128-35.

36. Boetius A, Ravenschlag K, Schubert CJ, Rickert D, Widdel F, Gieseke A, Amann R, Jørgensen BB, Witte U, Pfannkuche O. A marine microbial consortium apparently mediating anaerobic oxidation of methane. Nature. 2000;407:623-6.

37. Jones PR. Genetic instability in cyanobacteria—an elephant in the room? Front Bioeng Biotechnol. 2014;2:12.

38. Ducat DC, Avelar-Rivas JA, Way JC, Silver PA. Rerouting carbon flux to enhance photosynthetic productivity. Appl Environ Microbiol. 2012;78:2660-8.

39. Smith MJ, Francis MB. A designed A. vinelandii-S. elongatus coculture for chemical photoproduction from air, water, phosphate, and trace metals. ACS Synth Biol. 2016;5:955-61.

40. Hays SG, Yan LLW, Silver PA, Ducat DC. Synthetic photosynthetic consortia define interactions leading to robustness and photoproduction. J Biol Eng. 2017;11:4.

41. Weiss TL, Young EJ, Ducat DC. A synthetic, light-driven consortium of cyanobacteria and heterotrophic bacteria enables stable polyhydroxybutyrate production. Metab Eng. 2017:44:236-45.

42. Li T, Li CT, Butler K, Hays SG, Guarnieri MT, Oyler GA, Betenbaugh MJ. Mimicking lichens: incorporation of yeast strains together with sucrose-secreting cyanobacteria improves survival, growth, ROS removal, and lipid production in a stable mutualistic co-culture production platform. Biotechnol Biofuels. 2017;10:55.

43. Bohutskyi P, Kucek LA, Hill E, Pinchuk GE, Mundree SG, Beliaev AS. Conversion of stranded waste-stream carbon and nutrients into valueadded products via metabolically coupled binary heterotroph-photoautotroph system. Bioresour Technol. 2018;260:68-75.

44. Higgins BT, Labavitch JM, VanderGheynst JS. Co-culturing Chlorella minutissima with Escherichia coli can increase neutral lipid production and improve biodiesel quality. Biotechnol Bioeng. 2015;112:1801-9.

45. Zhuang WQ, Yi S, Bill M, Brisson VL, Feng X, Men Y, Conrad ME, Tang YJ, Alvarez-Cohen L. Incomplete Wood-Ljungdahl pathway facilitates one-carbon metabolism in organohalide-respiring Dehalococcoides mccartyi. Proc Natl Acad Sci USA. 2014;111:6419-24.

46. Yang WXH. Industrial fermentation of vitamin C. In: Vandamme ERJ, editor. Industrial biotechnology of vitamins, biopigments, and antioxidants. Weinheim: Wiley; 2016.

47. Song H, Ding MZ, Jia XQ, Ma Q, Yuan YJ. Synthetic microbial consortia: from systematic analysis to construction and applications. Chem Soc Rev. 2014;43:6954-81.

48. Chang MCY, Eachus RA, Trieu W, Ro DK, Keasling JD. Engineering Escherichia coli for production of functionalized terpenoids using plant P450s. Nat Chem Biol. 2007;3:274-7.

49. Chen X, Gao C, Guo L, Hu G, Luo Q, Liu J, Nielsen J, Chen J, Liu L. DCEO biotechnology: tools to design, construct, evaluate, and optimize the metabolic pathway for biosynthesis of chemicals. Chem Rev. 2018;118:4-72

50. Shou W, Ram S, Vilar JMG. Synthetic cooperation in engineered yeast populations. Proc Natl Acad Sci. 2007;104:1877-82.

51. Zhou K, Zou R, Stephanopoulos G, Too HP. Metabolite profiling identified methylerythritol cyclodiphosphate efflux as a limiting step in microbial isoprenoid production. PLoS ONE. 2012;7:e47513.

52. Abernathy MH, He L, Tang YJ. Channeling in native microbial pathways: implications and challenges for metabolic engineering. Biotechnol Adv. 2017;35:805-14.

53. Wolfsberg E, Long CP, Antoniewicz MR. Metabolism in dense microbial colonies: ${ }^{13} \mathrm{C}$ metabolic flux analysis of E. coli grown on agar identifies two distinct cell populations with acetate cross-feeding. Metab Eng. 2018;49:242-7.

54. Bruce JB, Cooper GA, Chabas H, West SA, Griffin AS. Cheating and resistance to cheating in natural populations of the bacterium Pseudomonas fluorescens. Evolution. 2017;71:2484-95.

55. Xiao Y, Bowen CH, Liu D, Zhang F. Exploiting nongenetic cell-to-cell variation for enhanced biosynthesis. Nat Chem Biol. 2016;12:339-44.

56. Xiu Y, Jang S, Jones JA, Zill NA, Linhardt RJ, Yuan Q, Jung GY, Koffas MAG. Naringenin-responsive riboswitch-based fluorescent biosensor module for Escherichia coli co-cultures. Biotechnol Bioeng. 2017;114:2235-44.
57. Li H, Opgenorth PH, Wernick DG, Rogers S, Wu TY, Higashide W, Malati P, Huo YX, Cho KM, Liao JC. Integrated electromicrobial conversion of $\mathrm{CO}_{2}$ to higher alcohols. Science. 2012;335:1596.

58. Morrison CS, Armiger WB, Dodds DR, Dordick JS, Koffas MAG. Improved strategies for electrochemical 1,4-NAD(P)H2 regeneration: a new era of bioreactors for industrial biocatalysis. Biotechnol Adv. 2018;36:120-31.

59. Kornienko N, Sakimoto KK, Herlihy DM, Nguyen SC, Alivisatos AP, Harris CB, Schwartzberg A, Yang P. Spectroscopic elucidation of energy transfer in hybrid inorganic-biological organisms for solar-to-chemical production. Proc Natl Acad Sci USA. 2016;113:11750-5.

60. Sakimoto KK, Wong AB, Yang P. Self-photosensitization of nonphotosynthetic bacteria for solar-to-chemical production. Science. 2016;351:74-7.

61. LaSarre B, McCully AL, Lennon JT, McKinlay JB. Microbial mutualism dynamics governed by dose-dependent toxicity of cross-fed nutrients. ISME J. 2017;11:337-48.

62. McCully AL, LaSarre B, McKinlay JB. Growth-independent cross-feeding modifies boundaries for coexistence in a bacterial mutualism. Environ Microbiol. 2017;19:3538-50.

63. Tang J. Microbial metabolomics. Curr Genomics. 2011;12:391-403.

64. Boghigian BA, Seth G, Kiss R, Pfeifer BA. Metabolic flux analysis for pharmaceutical production. Metab Eng. 2010;12:81-95.

65. Schuetz R, Kuepfer L, Sauer U. Systematic evaluation of objective functions for predicting intracellular fluxes in Escherichia coli. Mol Syst Biol. 2007;3:119.

66. Burgard AP, Pharkya P, Maranas CD. Optknock: a bilevel programming framework for identifying gene knockout strategies for microbial strain optimization. Biotechnol Bioeng. 2003;84:647-57.

67. Xu P, Ranganathan S, Fowler ZL, Maranas CD, Koffas MAG. Genomescale metabolic network modeling results in minimal interventions that cooperatively force carbon flux towards malonyl-CoA. Metab Eng. 2011;13:578-87.

68. Chemler JA, Fowler ZL, McHugh KP, Koffas MAG. Improving NADPH availability for natural product biosynthesis in Escherichia coli by metabolic engineering. Metab Eng. 2010;12:96-104.

69. Koch S, Benndorf D, Fronk K, Reichl U, Klamt S. Predicting compositions of microbial communities from stoichiometric models with applications for the biogas process. Biotechnol Biofuels. 2016;9:17.

70. Milne CB, Kim PJ, Eddy JA, Price ND. Accomplishments in genome-scale in silico modeling for industrial and medical biotechnology. Biotechnol J. 2009:4:1653-70.

71. Miller IJ, Vanee N, Fong SS, Lim-Fong GE, Kwan JC. Lack of overt genome reduction in the bryostatin-producing bryozoan symbiont "Candidatus Endobugula sertula". Appl Environ Microbiol. 2016;82:6573-83.

72. Salimi F, Zhuang K, Mahadevan R. Genome-scale metabolic modeling of a clostridial co-culture for consolidated bioprocessing. Biotechnol J. 2010;5:726-38.

73. Gomez JA, Höffner K, Barton PI. From sugars to biodiesel using microalgae and yeast. Green Chem. 2016;18:461-75.

74. Eng A, Borenstein E. An algorithm for designing minimal microbial communities with desired metabolic capacities. Bioinformatics. 2016;32:2008-16.

75. Julien-Laferrière A, Bulteau L, Parrot D, Marchetti-Spaccamela A, Stougie L, Vinga S, Mary A, Sagot MF. A combinatorial algorithm for microbial consortia synthetic design. Sci Rep. 2016;6:29182.

76. Magnúsdóttir S, Heinken A, Kutt L, Ravcheev DA, Bauer E, Noronha A, Greenhalgh K, Jäger C, Baginska J, Wilmes P, Fleming RMT, Thiele I. Generation of genome-scale metabolic reconstructions for 773 members of the human gut microbiota. Nat Biotechnol. 2016;35:81-9.

77. Karr JR, Sanghvi JC, Macklin DN, Gutschow MV, Jacobs JM, Bolival B, Assad-Garcia N, Glass Jl, Covert MW. A whole-cell computational model predicts phenotype from genotype. Cell. 2012;150:389-401.

78. Thiele I, Fleming RMT, Que R, Bordbar A, Diep D, Palsson BO. Multiscale modeling of metabolism and macromolecular synthesis in E. coli and its application to the evolution of codon usage. PLoS ONE. 2012;7:e45635.

79. You L, Liu H, Blankenship RE, Tang YJ. Using photosystem I as a reporter protein for ${ }^{13} \mathrm{C}$ analysis in a coculture containing cyanobacterium and a heterotrophic bacterium. Anal Biochem. 2015;477:86-8.

80. Shaikh AS, Tang YJ, Mukhopadhyay A, Keasling JD. Isotopomer distributions in amino acids from a highly expressed protein as a proxy for those from total protein. Anal Chem. 2008;80:886-90. 
81. Rühl M, Hardt WD, Sauer U. Subpopulation-specific metabolic pathway usage in mixed cultures as revealed by reporter protein-based ${ }^{13} \mathrm{C}$ analy sis. Appl Environ Microbiol. 2011;77:1816-21.

82. Ghosh A, Nilmeier J, Weaver D, Adams PD, Keasling JD, Mukhopadhyay A, Petzold CJ, Martin HG. A peptide-based method for ${ }^{13} \mathrm{C}$ metabolic flux analysis in microbial communities. PLoS Comput Biol. 2014;10:e1003827.
83. Gebreselassie NA, Antoniewicz MR. (13)C-metabolic flux analysis of cocultures: a novel approach. Metab Eng. 2015;31:132-9.
Ready to submit your research? Choose BMC and benefit from:

- fast, convenient online submission

- thorough peer review by experienced researchers in your field

- rapid publication on acceptance

- support for research data, including large and complex data types

- gold Open Access which fosters wider collaboration and increased citations

- maximum visibility for your research: over $100 \mathrm{M}$ website views per year

At BMC, research is always in progress.

Learn more biomedcentral.com/submissions 